\title{
Influência da temperatura na mortalidade de imaturos de Centris (Heterocentris) analis (Hymenoptera, Apidae, Centridini)
}

\author{
Ricardo Marques Couto \& Evandro Camillo
}

Departamento de Biologia, Faculdade de Filosofia, Ciências e Letras de Ribeirão Preto, USP, Av. Bandeirantes, 3900, $14040-901$ Ribeirão Preto, São Paulo, Brasil. (rima@pg.ffclrp.usp.br)

\begin{abstract}
Influence of temperature on the immatures mortality of Centris (Heterocentris) analis (Hymenoptera, Apidae, Centridini). Centris (Heterocentris) analis (Fabricius, 1804) nests were studied at the University Campus of Ribeirão Preto, São Paulo, Brazil, from January to December 2003. The females constructed nests in trap-nests made with black cardboard introduced in wood blocks which were placed on the shelves of two shelters built at the study site: sunny area and shady area. In both areas, the immatures mortality occurred mainly in the egg stage; shady area: egg stage (76.7\%), larval stage (10.0\%) and pupal stage (13.3\%); sunny area: egg stage $(80.0 \%)$, larval stage $(6.6 \%)$ and pupal stage $(13.3 \%)$. The mortality difference between the areas $\left(\chi^{2}=1.0\right.$, gl:1, p $\left.>0.05\right)$ was not great enough to reject the possibility that the difference was due to random sampling variability, however, there were differences of temperature between two areas in all months observed (t-student, $\mathrm{p}<0.05$ ) and the temperature around $26-27^{\circ} \mathrm{C}$ showed to be optimal to Centris analis development.
\end{abstract}

KEYWORDS. Hymenoptera, Centris, temperature, immatures mortality, development.

RESUMO. Os ninhos de Centris (Heterocentris) analis (Fabricius, 1804) foram estudados no Campus de Ribeirão Preto da Universidade de São Paulo, de janeiro a dezembro de 2003. As fêmeas nidificaram em ninhos-armadilha confeccionados com cartolina preta, os quais foram introduzidos em placas de madeira colocadas em duas áreas distintas: sombreada e ensolarada. Nas duas áreas, a mortalidade de imaturos ocorreu principalmente no estágio de ovo; área sombreada: estágio de ovo (76,7\%), estágio de larva (10,0\%) e estágio de pupa (13,3\%); área ensolarada: estágio de ovo $(80,0 \%)$, estágio de larva $(6,6 \%)$ e estágio de pupa (13,3\%). A diferença de mortalidade entre as duas áreas $\left(\chi^{2}=1,0\right.$, gl:1, p>0,05) não foi estatisticamente significativa, entretanto, a diferença de temperatura entre os meses nas duas áreas estudadas foi estatisticamente significativa (t-student, $\mathrm{p}<0,05$ ). Os resultados mostraram também que a temperatura entre $26^{\circ} \mathrm{C}$ e $27^{\circ} \mathrm{C}$ foi ideal para o desenvolvimento de Centris analis.

PALAVRAS-CHAVE. Hymenoptera, Centris, mortalidade de imaturos, temperatura, desenvolvimento.

A adaptação, o desenvolvimento e a sobrevivência dos organismos ectotérmicos são altamente dependentes do ambiente térmico (Sharpe \& DeMichele, 1977; WaGner \& Coulson, 1984; Gilchrist, 1995; Liu et al., 1995). O desenvolvimento dos imaturos de abelhas é influenciado pelas temperaturas elevadas, as quais podem provocar altas taxas de mortalidade, principalmente nos primeiros estádios larvais (ARRETZ, 1973; UNDURRAGA \& STEPHEN, 1980; FrANKIE et al., 1993).

Taxas de mortalidade têm sido determinadas em inúmeras espécies de abelhas que nidificam em cavidades preexistentes. Estas taxas variam de 3,9\% a 62,6\%, sendo atribuídas a causas desconhecidas que ocasionam falhas no desenvolvimento, observadas em Osmia marginata (Michener, 1936) (TEPEDINO \& PARKER, 1983), Hoplitis fulgida (Cresson, 1864) (TEPEDINO \& PARKER, 1984), Osmia sanrafaelae Parker, 1985 (PARKER, 1986), Centris vittata Lepeletier, 1841 (PEREIRA et al., 1999), C. analis (Fabricius, 1804) (Jesus \& GaróFalo, 2000; Gazola \& GaróFALO, 2003) e C. tarsata Smith, 1874 (Aguiar \& GARÓFALO, 2004). Em Megachile rotundata (Fabricius, 1787), a exposição à temperatura de $45^{\circ} \mathrm{C}$ não causou mortalidade significativa nas pupas e pré-pupas; no entanto, na exposição a $50^{\circ} \mathrm{C}$, a mortalidade foi total (UndurRaga \& STHEPHEN, 1980). PARKer (1986) observou em $O$. sanrafaelae que a alta taxa de mortalidade nas fases iniciais de desenvolvimento pode ter sido devido à temperatura, pois os blocos de madeira menores $(\varnothing=6 \mathrm{~mm}$ ) que continham os ninhos poderiam absorver e reter mais calor que os maiores $(\varnothing=$ $9 \mathrm{~mm}$ ), tornando-se, assim, letais para os imaturos. Segundo Frankie et al. (1988), abelhas do gênero Centris nidificam, preferencialmente, em ambientes sombreados e temperaturas entre $38^{\circ} \mathrm{C} \mathrm{e} 40^{\circ} \mathrm{C}$ seriam letais para larvas de $C$. analis. Altas taxas de mortalidade encontradas em ovos e larvas jovens de $C$. analis podem ser causadas por elevadas temperaturas nos locais de nidificação, as quais afetariam somente os estágios iniciais de desenvolvimento (Jesus \& Garófalo, 2000; Gazola \& GARÓFALO, 2003).

O gênero Centris é originalmente tropical, cujas espécies estão distribuídas em 12 subgêneros. A maioria das espécies constroem os ninhos no solo enquanto que aquelas pertencentes aos subgêneros Hemisiella, Heterocentris e Xanthemisia nidificam em cavidades preexistentes (Coville et al., 1983, 1986; FrankiE et al., 1988, 1989, 1993). Centris analis é solitária, ocorrendo do México ao Brasil (Moure, 1960).

Segundo Jesus \& Garófalo (2000), fêmeas de $C$. analis construíram seus ninhos em ninhos-armadilha com material vegetal e uma substância oleosa. Ninhos completos apresentaram de 1 a 4 células arranjadas em série linear, usualmente seguidas por uma célula vestibular, uma célula vazia localizada entre a última célula de cria e o fechamento do ninho. Machos foram criados nas células mais externas enquanto que as fêmeas naquelas mais internas. O período de desenvolvimento foi afetado 
pelas condições climáticas e variou de 36 a 85 dias para os machos e de 37 a 88 dias para as fêmeas. Ninhos foram parasitados pela vespa Leucospis cayennensis Westwood, 1839 e pelas abelhas Coelioxys sp. e Mesocheira bicolor (Fabricius, 1804).

O objetivo deste trabalho é apresentar dados relacionados à influência da temperatura no desenvolvimento e na taxa de mortalidade dos imaturos de $C$. analis.

\section{MATERIAL E MÉTODOS}

O estudo foi realizado no Campus da Universidade de São Paulo, município de Ribeirão Preto, São Paulo $\left(21^{\circ} 05^{\prime}-21^{\circ} 15^{\prime} \mathrm{S}\right.$ e $\left.47^{\circ} 50^{\prime}-47^{\circ} 55^{\prime} \mathrm{W}\right)$. A área possui aproximadamente 573 ha, com altitudes entre 530 e $675 \mathrm{~m}$. O solo é caracterizado como latossolo roxo eutrófico, com textura argilosa ou muito argilosa (OliveIRA \& PRADO, 1987). Na área de estudo, pouco resta da vegetação nativa; atualmente está composta por gramíneas, ervas, arbustos e um reflorestamento com exemplares de espécies nativas e exóticas. De acordo com as condições climáticas (temperatura e precipitação), ocorrem duas estações na área de estudo: uma fria e seca (maio a agosto) e uma quente e chuvosa (setembro a abril).

Os ninhos-armadilha (NA) utilizados no estudo foram confeccionados com cartolina preta na forma de pequenos tubos, com uma das extremidades fechada com o mesmo material, apresentando 0,6 cm de diâmetro e 5,8 $\mathrm{cm}$ de comprimento. Estes NA $(\mathrm{n}=330)$ foram introduzidos em orifícios feitos em placas de madeira de $5 \mathrm{~cm}$ de largura, $30 \mathrm{~cm}$ de comprimento e $12 \mathrm{~cm}$ de altura com 55 orifícios por placa $(n=6)$. As placas foram colocadas em prateleiras construídas sob duas áreas distintas: sombreada e ensolarada, próximas ao Laboratório de Ecologia do Departamento de Biologia da Faculdade de Filosofia, Ciências e Letras de Ribeirão Preto, SP-USP.

Cada área foi caracterizada por um abrigo de $2 \mathrm{~m}$ de largura, $3 \mathrm{~m}$ de comprimento e $2 \mathrm{~m}$ de altura, coberto por telhas de amianto. Na área sombreada o abrigo estava localizado sob a copa de uma mangueira (Mangifera sp., Anacardiaceae), a qual forneceu sombra durante o dia todo, enquanto que na área ensolarada o abrigo ficou exposto diretamente ao sol durante o dia. A distância entre as duas áreas (abrigos) foi de $10 \mathrm{~m}$ e a investigação ocorreu durante o ano de 2003, com observações diárias entre 8:00 e 17:00h. A coleta de dados baseou-se nas observações das atividades de nidificação e mensuração da temperatura. Durante a nidificação, foram registradas as datas de início e término da construção de cada célula.

Para a obtenção de dados sobre temperatura do ninho, foram utilizados dois relógios termo-higrômetros, localizados um em cada área, cujos sensores foram colocados em contato com as placas de madeira contendo os mesmos. As nidificações foram acompanhadas com o auxílio de um otoscópio, útil para a observação no interior do ninho. Vinte dias após o fechamento dos ninhos, os mesmos foram transferidos para um tubo de vidro, o qual foi fechado com uma rolha de cortiça com o objetivo de se observar a emergência e o sexo dos indivíduos. Alguns dias após a emergência, os ninhos foram abertos no laboratório para se obter a taxa de mortalidade nos estágios de ovo, larva ou pupa.

A razão sexual e as possíveis diferenças nas taxas de mortalidade entre as áreas sombreada e ensolarada, bem como entre seus respectivos meses, foram analisadas pelo teste do $\chi^{2}$ (Qui-Quadrado). A temperatura e o tempo de desenvolvimento foram analisados pelo programa Sigmastat Statistical, que disponibilizou os testes de Tukey, Dann's e o t-student.

\section{RESULTADOS}

Número de ninhos e mortalidade. Indivíduos de Centris analis construíram 81 ninhos com 215 células, das quais em 90 não foram verificadas emergências $(41,8 \%)$. As nidificações ocorreram nos meses de setembro a maio, sendo janeiro e fevereiro da área ensolarada, e janeiro e outubro da área sombreada, os meses que apresentaram o maior número delas (Fig. 1).

Das células onde não ocorreram emergências, 71 delas apresentaram mortalidade no estágio de ovo $(78,9 \%)$, sete no estágio de larva $(7,8 \%)$ e 12 no estágio de pupa $(13,3 \%)$ (Tab. I). A razão sexual foi de 74 machos: 51 fêmeas, a qual é diferente de $1: 1\left(\chi^{2}=4,2, \mathrm{gl}: 1, \mathrm{p}<0,05\right)$.

Área sombreada. Nesta área foram construídos 29 ninhos com um total de 80 células, das quais em 30 não

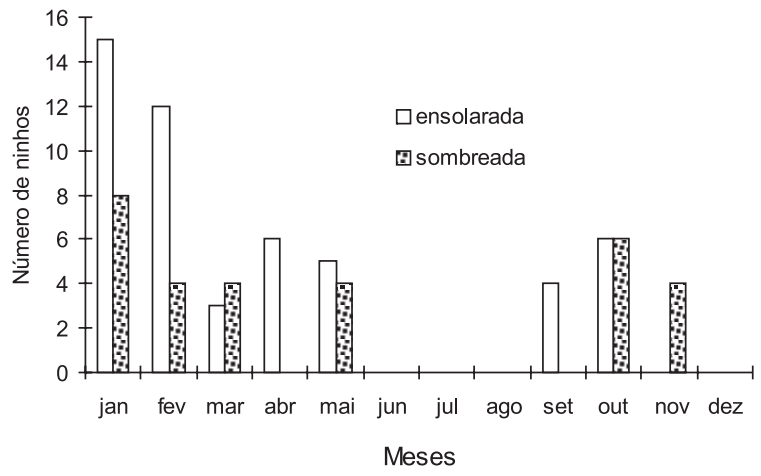

Fig. 1. Número mensal de ninhos de Centris (Heterocentris) analis construídos durante o período de janeiro a dezembro de 2003 nas áreas sombreada e ensolarada no campus da USP, Ribeirão Preto, SP.

Tabela I. Dados de Centris (Heterocentris) analis obtidos nas áreas sombreada e ensolarada no período de janeiro e dezembro de 2003 no campus da USP, Ribeirão Preto. Valores seguidos por diferentes letras na mesma coluna diferem estatisticamente.

\begin{tabular}{lrrrrrrr}
\hline Meses & $\begin{array}{c}N^{\text {o }} \text { de } \\
\text { células }\end{array}$ & $\begin{array}{c}\text { Emer- } \\
\text { gências }\end{array}$ & $\begin{array}{c}\text { Não- } \\
\text { emer- } \\
\text { gências }\end{array}$ & $\begin{array}{c}\text { Mortali- } \\
\text { dade (\%) }\end{array}$ & \multicolumn{2}{c}{ Mortalidade } \\
\cline { 6 - 8 } Jan & 45 & 31 & 14 & $31,1 \mathrm{a}$ & 10 & 3 & 1 \\
Fev & 52 & 20 & 32 & $61,5 \mathrm{~b}$ & 23 & 3 & 6 \\
Mar & 20 & 5 & 15 & $75,0 \mathrm{~b}$ & 14 & - & 1 \\
Abr & 19 & 6 & 13 & $68,4 \mathrm{~b}$ & 11 & - & 2 \\
Mai & 20 & 10 & 10 & $50,0 \mathrm{ab}$ & 9 & 1 & - \\
Set & 12 & 7 & 5 & $41,6 \mathrm{ab}$ & 4 & - & 1 \\
Out & 34 & 33 & 1 & $2,9 \mathrm{c}$ & - & - & 1 \\
Nov & 13 & 13 & 0 & $0 \mathrm{c}$ & - & - & - \\
Total & 215 & 125 & 90 & 41,8 & 71 & 7 & 12 \\
\hline
\end{tabular}


foram verificadas emergências (37,5\%). Dentre estas, em 23 a mortalidade ocorreu no estágio de ovo $(76,7 \%)$, três no estágio de larva $(10,0 \%)$ e quatro no estágio de pupa (13,3\%). A razão sexual foi de 29 machos: 21 fêmeas, a qual foi semelhante a 1:1 $\left(\chi^{2}=1,3, \mathrm{gl}: 1, \mathrm{p}>0,05\right)$.

Nos meses que ocorreu oviposição, a taxa de mortalidade foi diferente estatisticamente $\left(\chi^{2}=32,2, \mathrm{gl}: 5\right.$, p $<0,05$ ), variando de $0 \%$ (outubro e novembro) a $81,8 \%$ em março. Entretanto, esses meses apresentaram temperatura média semelhante (Tab. II).

Nas oviposições que ocorreram em janeiro e outubro, o tempo de desenvolvimento dos machos foi similar. Para as fêmeas, o mesmo ocorreu nestes meses, porém outubro foi semelhante também a novembro (Tab. III). O tempo de desenvolvimento das fêmeas foi significativamente maior que o dos machos, na área sombreada, apenas no mês de outubro ( $\mathrm{t}=5,7, \mathrm{gl}: 16, \mathrm{p}<0,05)$.

Área ensolarada. Nesta área as fêmeas de C. analis construíram 52 ninhos e 135 células, nas quais não ocorreu emergência em 60 delas (44,5\%). Dentre estas, em 48 células a mortalidade ocorreu no estágio de ovo $(80,0 \%)$, quatro no estágio de larva $(6,6 \%)$ e oito no estágio de pupa (13,3\%) (Tab. IV). A razão sexual foi de 45 machos: 30 fêmeas, a qual foi semelhante a $1: 1\left(\chi^{2}=\right.$ $3,0, \mathrm{gl}: 1, \mathrm{p}>0,05)$.

A taxa de mortalidade mensal foi diferente estatisticamente $\left(\chi^{2}=27,5, \mathrm{gl}: 6, \mathrm{p}<0,05\right)$, variando de $6,2 \%$ (outubro) até $68,4 \%$ (abril). Em todos os meses, exceto maio, a temperatura média mensal foi semelhante, porém a taxa de mortalidade foi diferente (Tab. IV).
Em oviposições que ocorreram nos meses de janeiro, fevereiro, setembro e outubro, o tempo de desenvolvimento das fêmeas foi semelhante. Para os machos, nesses mesmos meses, apenas as oviposições ocorridas em janeiro e fevereiro foram diferentes (Tab. III). A diferença do tempo de desenvolvimento entre fêmeas e machos foi significativa apenas no mês de janeiro $(\mathrm{t}=5,4, \mathrm{gl}: 24, \mathrm{p}<0,05)$.

Sombreada X Ensolarada. A taxa de mortalidade obtida na área sombreada $(37,5 \%)$ não foi estatisticamente diferente $\left(\chi^{2}=1,0\right.$, gl: $\left.1, \mathrm{p}>0,05\right)$ daquela observada na área ensolarada $(44,5 \%)$. Entre os meses estudados, janeiro foi o único que apresentou diferença de mortalidade em relação aos outros $\left(\chi^{2}=5,65, \mathrm{gl}: 1, \mathrm{p}<0,05\right)$. Entretanto, a temperatura média mensal apresentou diferença significativa em todos os meses observados $(p<0,05)$.

Quando comparamos o tempo de desenvolvimento dos machos das duas áreas, notamos que ocorreu diferença significativa apenas no mês de outubro $(\mathrm{t}=$ 11,96, gl:11, p <0,001), enquanto que para as fêmeas ocorreu diferença nos meses de fevereiro $(\mathrm{t}=4,4, \mathrm{gl}: 7$, $p<0,05)$ e outubro $(t=6,4, g l: 11, p<0,001)$. O mês de janeiro não mostrou diferença quanto ao tempo de desenvolvimento dos machos $(\mathrm{t}=1,78, \mathrm{gl}: 8, \mathrm{p}>0,05) \mathrm{e}$ das fêmeas $(\mathrm{t}=2,0, \mathrm{gl}: 19, \mathrm{p}>0,05)$. O período de março a maio não foi considerado na análise estatística de tempo de desenvolvimento porque, nas nidificações que ocorreram nesta fase, os imaturos entraram em diapausa devido à baixa temperatura, dificultando a análise da influência da temperatura elevada sobre o desenvolvimento de C. analis.

Tabela II. Número de ninhos, células, emergências e mortalidade de imaturos de Centris (Heterocentris) analis na área sombreada e temperaturas médias $\left({ }^{\circ} \mathrm{C}\right)$ registradas. Valores sobre mortalidade $(\%)$ seguidos por letras diferentes na mesma coluna diferem estatisticamente. Valores sobre temperatura média acompanhados por letras diferentes na mesma coluna diferem estatisticamente.

\begin{tabular}{lccccccccc}
\hline \multirow{2}{*}{ Meses } & $\mathrm{N}^{\mathbf{o}}$ de ninhos & $\mathrm{N}^{\mathbf{o}}$ de células & Emergências & Mortalidade & $\begin{array}{c}\text { Mortalidade } \\
(\%)\end{array}$ & $\begin{array}{c}\text { Temperatura } \\
\text { média }\end{array}$ & \multicolumn{3}{c}{ Mortalidade } \\
\cline { 7 - 10 } & & & & & & & Ovo & Larva & Pupa \\
\hline Jan & 5 & 12 & 5 & 7 & $58,3 \mathrm{ab}$ & $27,0 \pm 1,3 \mathrm{a}$ & 5 & 2 & - \\
Fev & 6 & 16 & 6 & 10 & $62,5 \mathrm{ab}$ & $30,6 \pm 2,8 \mathrm{~b}$ & 6 & 1 & 3 \\
Mar & 4 & 11 & 2 & 9 & $81,8 \mathrm{a}$ & $26,7 \pm 2,7 \mathrm{a}$ & 8 & - & 1 \\
Mai & 4 & 10 & 6 & 4 & $40,0 \mathrm{~b}$ & $23,6 \pm 3,2 \mathrm{c}$ & 4 & - & - \\
Out & 6 & 18 & 18 & 0 & $0,0 \mathrm{c}$ & $27,0 \pm 2,9 \mathrm{a}$ & - & - & - \\
Nov & 4 & 13 & 13 & 0 & $0,0 \mathrm{c}$ & $25,9 \pm 1,2 \mathrm{a}$ & - & - & - \\
Total & 29 & 80 & 50 & 30 & 37,5 & - & 23 & 3 & 4 \\
\hline
\end{tabular}

Tabela III. Análise do tempo de desenvolvimento (em dias) dos imaturos de mesmo sexo de Centris (Heterocentris) analis nas áreas sombreada e ensolarada, campus da USP, Ribeirão Preto, SP. Valores seguidos por letras diferentes na mesma coluna (entre parêntesis) ou na mesma linha diferem estatisticamente.

\begin{tabular}{|c|c|c|c|c|}
\hline \multirow{2}{*}{ Meses } & \multicolumn{2}{|c|}{ Área sombreada } & \multicolumn{2}{|c|}{ Área ensolarada } \\
\hline & Macho (n) & Fêmea (n) & Macho (n) & Fêmea (n) \\
\hline Jan & $50,0 \pm 0,0(\mathrm{a}) \mathrm{a}(2)$ & $54,0 \pm 1,0(\mathrm{a}) \mathrm{a}(3)$ & $52,3 \pm 1,6(a) a(19)$ & $56,9 \pm 2,6(\mathrm{a}) \mathrm{a}(7)$ \\
\hline Fev & $60,6 \pm 1,5(\mathrm{~b}) \mathrm{a}(3)$ & $64,0 \pm 2,0(\mathrm{~b}) \mathrm{a}(3)$ & $54,1 \pm 3,4(\mathrm{~b}) \mathrm{a}(8)$ & $56,0 \pm 2,7(a) b(6)$ \\
\hline Mar & $114,0(\mathrm{c})(1)$ & $122,0(\mathrm{c})(1)$ & $112,0 \pm 0,0(\mathrm{c})(2)$ & $115,0(b)(1)$ \\
\hline Abr & - & - & $110,6 \pm 2,5(\mathrm{c})(3)$ & $113,3 \pm 1,5(b)(3)$ \\
\hline Mai & $113,5 \pm 1,5(\mathrm{c}) \mathrm{a}(2)$ & $114,7 \pm 1,3(\mathrm{c}) \mathrm{a}(4)$ & $115,5 \pm 0,7(\mathrm{c}) \mathrm{a}(2)$ & $118,0 \pm 1,4(\mathrm{~b}) \mathrm{a}(2)$ \\
\hline Set & - & - & $55,5 \pm 0,6(a b)(4)$ & $58,0 \pm 1,0(a)(3)$ \\
\hline Out & $46,7 \pm 1,2(a) a(13)$ & $50,2 \pm 0,8(\mathrm{ad}) \mathrm{a}(5)$ & $53,7 \pm 1,2(a b) b(7)$ & $53,7 \pm 1,2(a) b(8)$ \\
\hline Nov & $44,1 \pm 2,7(\mathrm{~d})(8)$ & $47,0 \pm 2,5(\mathrm{~d})(5)$ & - & - \\
\hline
\end{tabular}


Tabela IV. Número de ninhos, células, emergências e mortalidade de imaturos de Centris (Heterocentris) analis na área ensolarada no campus da USP, Ribeirão Preto, SP e temperaturas médias $\left({ }^{\circ} \mathrm{C}\right)$ registradas. Valores sobre mortalidade $(\%)$ seguidos por letras diferentes na mesma coluna diferem estatisticamente. Valores sobre temperatura média seguidos por letras na mesma coluna diferem estatisticamente.

\begin{tabular}{|c|c|c|c|c|c|c|c|c|c|}
\hline \multirow{2}{*}{ Meses } & \multirow{2}{*}{$\mathrm{N}^{\circ}$ de ninhos } & \multirow{2}{*}{$\mathrm{N}^{\mathrm{o}}$ de células } & \multirow[t]{2}{*}{ Emergências } & \multirow[t]{2}{*}{ Mortalidade } & \multirow{2}{*}{$\begin{array}{l}\text { Mortalidade } \\
(\%)\end{array}$} & \multirow{2}{*}{$\begin{array}{l}\text { Temperatura } \\
\text { média }\end{array}$} & \multicolumn{3}{|c|}{ Mortalidade } \\
\hline & & & & & & & Ovo & Larva & Pupa \\
\hline Jan & 15 & 33 & 26 & 7 & $21,2 \mathrm{ac}$ & $33,3 \pm 3,2 \mathrm{a}$ & 5 & 1 & 1 \\
\hline Fev & 13 & 36 & 14 & 22 & $61,1 b$ & $35,1 \pm 4,0 \mathrm{a}$ & 17 & 2 & 3 \\
\hline Mar & 3 & 9 & 3 & 6 & $66,6 b$ & $31,4 \pm 4,5 a$ & 6 & - & - \\
\hline Abr & 6 & 19 & 6 & 13 & $68,4 b$ & $30,7 \pm 4,0 \mathrm{a}$ & 11 & - & 2 \\
\hline Mai & 5 & 10 & 4 & 6 & $60,0 \mathrm{~b}$ & $26,8 \pm 3,4 b$ & 5 & 1 & \\
\hline Set & 4 & 12 & 7 & 5 & $41,6 a b$ & $31,4 \pm 4,5 a$ & 4 & - & 1 \\
\hline Out & 6 & 16 & 15 & 1 & $6,25 c$ & $32,2 \pm 3,7 \mathrm{a}$ & - & - & 1 \\
\hline Total & 52 & 135 & 75 & 60 & 44,5 & & 48 & 4 & 8 \\
\hline
\end{tabular}

\section{DISCUSSÃO}

Eves \& JOHANSEN (1974) observaram que a maior causa de mortalidade de ovos e larvas jovens em Megachile rotundata foi a temperatura elevada. PARKER \& Frohlich (1985) verificaram que, em Eumagechile pugnata (Say, 1837), a mortalidade de ovos e larvas recémeclodidas foi em torno de $30 \%$, ocasionada pela temperatura elevada no interior dos ninhos. MoRATO (2001) observou em Anthodioctes moratoi Urban, 1999 mortalidade em pelo menos uma célula em $52 \%$ dos ninhos analisados. AgUiar (2002) registrou, em ninhos de $C$. tarsata, taxas de mortalidade de imaturos entre $41 \mathrm{e}$ $42 \%$, ocorrendo o maior índice na fase pré-emergência.

A influência da temperatura no desenvolvimento dos imaturos de abelhas do gênero Centris foi avaliada por FRANKIE et al. (1988), e os resultados mostraram que elevadas temperaturas em condições naturais são causadoras de elevados índices de mortalidade nos primeiros estádios larvais. FrANKIE et al. (1993) observaram em laboratório que temperatura constante de $40^{\circ} \mathrm{C}$ afetou o desenvolvimento e causou mortalidade nos primeiros estádios larvais. Entretanto, no presente trabalho, os resultados mostraram que a mortalidade concentrou-se principalmente no estágio de ovo e que as taxas de mortalidade nas áreas sombreada e ensolarada foram semelhantes estatisticamente, embora a temperatura entre as duas áreas tenha sido diferente estatisticamente em todos os meses analisados.

Por outro lado, a temperatura em torno de $26-27^{\circ} \mathrm{C}$ pode ser considerada ótima para o desenvolvimento dos imaturos de $C$. analis, pois nos meses que apresentaram esta temperatura média (outubro e novembro), a taxa de mortalidade foi de $0 \%$. Apesar da diferença de temperatura entre os meses, nas duas áreas, o tempo de desenvolvimento dos imaturos de $C$. analis mostrou-se semelhante em janeiro para machos e fêmeas e fevereiro para os machos. A temperatura ideal na qual a mortalidade de imaturos foi de $0 \%$ e o tempo de desenvolvimento menor é resultado que poderá ser utilizado em projetos de polinização de algumas frutíferas cultivadas comercialmente.

A temperatura foi fator importante no desenvolvimento dos imaturos de $C$. analis, porém não o único. A alta taxa de mortalidade obtida no estágio de ovo, tanto na área sombreada como na ensolarada, sugere que o efeito maternal possa ter sido outro fator importante que interferiu na sobrevivência dos imaturos, como observado em algumas espécies de insetos por UvAROV (1961), Wellington (1965), LeONARd (1970), CAPINERA \& Barbosa (1976), GreEnblatt \& WitTER (1976), Rossiter (1991), Rossiter et al. (1993) e Fox \& Musseau (1998).

O efeito maternal é produto de uma interação entre fenótipo parental, ambiente parental, genótipo do descendente e ambiente do descendente (Rossiter, 1991). Segundo Fox \& Mousseau (1998), o efeito maternal modifica o tamanho dos ovos, estabelecendo uma quantidade de vitelogenina que está relacionada com a adaptação e fecundidade dos descendentes.

RossiTER et al. (1993) demonstraram que a condição nutricional parental tem uma influência significativa na magnitude da variação fenotípica do nível de vitelogenina armazenada nos ovos. Além da condição nutricional parental, a idade da fêmea é outro fator importante que modifica o tamanho dos ovos. De acordo com Fox \& Mousseau (1998), as fêmeas mais longevas apresentam uma menor taxa de fecundidade em relação às fêmeas mais novas, porém, quando o alimento é disponibilizado em abundância, as fêmeas mais longevas aumentam a taxa de fecundidade.

Esses dados apóiam a hipótese de que o efeito maternal resulta em uma grande diversidade fenotípica nos descendentes, contribuindo para o sucesso de espécies oportunistas. A produção de variação fenotípica pode ser útil em relação a um ambiente heterogêneo e imprevisível (KAPLAN \& COOPER, 1984). Os dados disponíveis na literatura sobre o efeito maternal estão concentrados principalmente em Lepidoptera e Coleoptera, que apresentam, como nos insetos da ordem Hymenoptera, cuidado parental na escolha do alimento para os descendentes. Sendo assim, este trabalho discute e sugere que o conceito de efeito maternal possa ser utilizado como um modelo para os himenópteros e para outros insetos holometábolos.

Agradecimentos. Ao apoio técnico de José Carlos Serrano e às sugestões do Dr. Carlos Alberto Garófalo, da Faculdade de Filosofia Ciências e Letras de Ribeirão Preto, Universidade de São Paulo. 


\section{REFERÊNCIAS BIBLIOGRÁFICAS}

Aguiar, C. M. L. 2002. Abelhas (Hymenoptera, Apoidea) que nidificam em ninhos-armadilha, em áreas de caatinga e floresta estacional semi-decídua do Estado da Bahia, Brasil. In: Encontro sobre abelhas, 5, Ribeirão Preto, 2002. Anais ... Ribeirão Preto, USP. p.53-57.

Aguiar, C. M. L. \& Garófalo, C. A. 2004. Nesting biology of Centris (Hemisiella) tarsata Smith (Hymenoptera, Apidae, Centridini). Revista Brasileira de Zoologia 21(3):1-9.

Arretz, P. 1973. Factores de mortalidad de Megachile rotundata (Fabricius) en Chile (Hymenoptera: Megachilidae). Revista Chilena de Entomologia 7:59-78.

Capinera, J. L. \& Barbosa, P. 1976. Dispersal of first-instar gypsy moth larval in relation to population quality. Oecologia 26:53-60.

Coville, R. E.; Frankie, G. W. \& Vinson, S. B. 1983. Nests of Centris segregata (Hymenoptera: Anthophoridae) with a review of the nesting habits of the genus. Journal of the Kansas Entomological Society 56:109-122.

Coville, R. E.; Frankie, G. W.; Buchmann, S. L.; Vinson, S. B. \& Willians, H. J. 1986. Nesting and male behavior of Centris heithausi in Costa Rica (Hymenoptera, Anthophoridae) with chemical analysis of the hind leg glands of males. Journal of the Kansas Entomological Society 59:325-336.

Eves, J. D. \& Johansen, C. A. 1974. Population dynamics of larvae leafcutting bee, Megachile rotundata, in eastern Washington. Washington Agricultural Experimental Station Bulletin 78:1-12.

Fox, C. W. \& Mousseau, T. A. 1998. The adaptive significance of maternal effects. Tree 13(10):403-407.

Frankie, G. W.; Vinson, S. B. \& Willians, H. 1989. Ecological and evolutionary sorting of 12 sympatric species of Centris bees in Costa Rican dry forest. In: Bock, J. H. \& Linhart, Y. B. eds. The Evolutionary Ecology of Plants. Boulder, Westview. p.535-549.

Frankie, G. W.; Newstrom, L.; Vinson, S. B. \& Barthell, J. F. 1993. Nesting-habitat preferences of selected Centris bee species in Costa Rican dry forest. Biotropica 25(3):322-333.

Frankie, G. W.; Vinson, S. B.; Newstrom, L. \& Barthell, J. F. 1988. Nest site habitat preferences of Centris bees in the Costa Rican dry forest. Biotropica 20(4):301-310.

Gazola, A. N. \& Garófalo, C. A. 2003. Parasitic behavior of Leucospis cayennensis Westwood (Hymenoptera, Leucospidae) and rates of parasitism in populations of Centris (Heterocentris) analis (Fabricius 1804) (Hymenoptera: Apidae: Centridini). Journal of the Kansas Entomological Society 76:131-142.

Gilchrist, G. W. 1995. Specialists and generalists in changing environments. Fitness landscapes of thermal sensitivity. American Naturalist 146(2):252-269.

Greenblatt, J. A. \& Witter, J. A. 1976. Behavioural studies on Malacosoma disstria (Lepidoptera: Lasiocampidae). Canadian Entomologist 108:1225-1228.

Jesus, B. M. \& Garófalo, C. A. 2000. Nesting behaviour of Centris (Heterocentris) analis (Fabricius) in southeastern Brazil (Hymenoptera, Apidae, Centridini). Apidologie 31:503-515.

Kaplan, R. H. \& Cooper, W. S. 1984. The evolution of developmental plasticity in reproductive characteristics: an application of the adaptative coin-flipping principle.
American Naturalist 123:393-410.

LEONARD, D. E. 1970. Intrinsic factors causing qualitative changes in population of Porthetria dispar (Lepidoptera: Lymantriidae). Canadian Entomologist 102:239-249.

LiU, S. S.; Zhang, G. M. \& ZHU, J. 1995. Influence of temperature variations on rate of development in insects: analyses of case studies from entomological literature. Annals of the Entomological Society of America 88(2):107-119.

Morato, E. F. 2001. Biologia e ecologia de Anthodioctes moratoi Urban (Hymenoptera, Megachilidae, Anthidiini) em matas contínuas e fragmentos na Amazônia Central, Brasil. Revista Brasileira de Zoologia 18(3):729-736.

Moure, J. S. 1960. Notes of types of the Neotropical bees described by Fabricius (Hymenoptera: Apoidea). Studia Entomologica 3:97-160.

Oliveira, J. B. \& Prado, H. 1987. Levantamento pedológico semidetalhado do Estado de São Paulo: quadrícula de Ribeirão Preto. II. Memorial descritivo. Campinas, Instituto Agronômico. 133p. (Boletim Científico, 7).

PARKER, F. D. 1986. Nesting, associates and mortality of Osmia sanrafaelae Parker. Journal of Kansas Entomological Society 59:367-377.

Parker, F. D. \& Frohlich, D. R. 1985. Studies on management of the sunflower leaf-cutter bee. Journal of Apicultural Research 24:125-131.

Pereira, M.; Garófalo, C. A.; Camillo, E. \& Serrano, J. C. 1999. Nesting biology of Centris (Hemisiella) vittata Lepeletier in southeastern Brazil (Hymenoptera, Apidae, Centridini). Apidologie 30:327-338.

Rossiter, M. C. 1991. Maternal effects generate variation in life history: consequences of egg weight plasticity in the gypsy moth. Functional Ecology 5(3):386-393.

Rossiter, M. C.; Cox-Foster, D. L. \& Briggs, M. A. 1993. Initiation of maternal effects in Lymantria dispar: genetic and ecological components of egg provisioning. Journal of Evolutionary Biology 6:577-589.

Sharpe, P. J. H. \& Demichele, D. V. 1977. Reaction kinetics of poikilotherm development. Journal of Theoretical Biology 64:649-670.

Tepedino, V. J. \& Parker, F. D. 1983. Nest size, mortality and sex ratio in Osmia marginata (Michener). The Southwestern Entomologist 8:154-166.

1984. Nest selection, mortality and sex ratio in Hoplitis fulgida (Cresson) (Hymenoptera: Megachilidae). Journal of Kansas Entomological Society 57:181-189.

Undurraga, J. M. \& Stephen, W. P. 1980. Effect of temperature on development and survival in post-diapausing alfafa leafcutting bee prepupae and pupae (Megachile rotundata (F)): (Hymenoptera: Megachilidae). I. High temperatures. Journal of Kansas Entomological Society 53:669-676.

Uvarov, B. P. 1961. Quantity and quality in insect populations. Proceedings of the Royal Entomological Society London 25:52-59.

Wagner, T. L. \& Coulson, R. N. 1984. Modeling insect development rates: a literature review and application of a biophysical model. Annals of the Entomological Society of America 77(2):208-225.

Wellington, W. G. 1965. Some maternal influences on progeny quality in the western caterpillar, Malacosoma pluviale (Dyar). Canadian Entomologist 97:1-14. 